Revista Perspectivas Online: Biológicas \& Saúde Abril de 2019, Vol.9, no 29, p.43- 55

ISSN: 2236-8868 (Online)

DOI: $10.25242 / 886892920191723$

\title{
INFORMAÇÃO SOBRE DENGUE ENTRE USUÁRIOS DA ESTRATÉGIA SAÚDE DA FAMÍLIA
}

\author{
Allen Cristina Ribeiro da Silva ${ }^{1}$, Katia de Oliveiro Melo ${ }^{1}$, Livian de Oliveira Silva ${ }^{1}$ \& Sinézio \\ Inácio da Silva Júnior ${ }^{2 *}$
}

\begin{abstract}
RESUMO
SILVA, A.C.R.; MELO, K.O. SILVA, L.O. \& JÚNIOR, S.I.S. Informação sobre dengue entre usuários da estratégia saúde da família Perspectivas Online: Biológicas \& Saúde. v. 9, n 29, p.43-55, 2019.

A dengue é uma doença reemergente no Brasil. Embora seja uma das prioridades do Pacto pela Vida, reincidentes epidemias vêm ocorrendo. Este estudo teve como objetivo coletar e analisar dados sobre o nível de informação a respeito da dengue entre usuários da Estratégia Saúde da Família (ESF) na cidade de Alfenas-MG, Brasil. É um estudo exploratório, quantitativo, descritivo e transversal. Foi realizada entrevista estruturada que foi aplicada a partir de uma amostragem por conveniência junto a 100 indivíduos distribuídos igualmente entre 10

ESF, no primeiro semestre de 2016. Foi realizada abordagem estatística descritiva e analítica com aplicação do teste exato de Fischer. Observou-se que a maioria tem informação sobre a doença, exceto sobre a forma hemorrágica. Os mais escolarizados demonstraram maior conhecimento sobre a dengue e sua prevenção, entretanto, foi entre eles que se observou o maior número de casos. Os resultados indicam a necessidade de estratégias inovadoras de educação em saúde para aumento de efetividade da política de prevenção.
\end{abstract}

Palavras-chave: Prevenção; Educação em saúde; Arbovirose. 


\begin{abstract}
Dengue fever is a reemerging disease in Brazil. While it is one of the priorities of the Pact for Life, recurrent epidemics have been occurring. This study aimed to collect and analyze data on the level of information on dengue among users of the Family Health Strategy (ESF) in the city of Alfenas-MG, Brazil. It is an exploratory, quantitative, descriptive and cross-sectional study. A structured interview was performed, which was applied from a convenience sample to 100 individuals equally distributed among 10

ESF in the first half of 2016. It was applied descriptive statistic and analytical approach using the Fisher exact test. The results indicated that most people have information about the disease, except on the hemorrhagic form. The more educated showed greater knowledge about dengue and its prevention, however, it was among them that the greatest number of cases of the disease was reported. The results indicate the need for innovative health education strategies to increase the effectiveness of prevention policy.
\end{abstract}

Keywords: Prevention; Health education; Arboviruses.

${ }^{1}$ Universidade Federal de Alfenas - Graduada em Farmácia - Rua Gabriel Monteiro da Silva, 700, CEP: 37.130-001, Alfenas-MG.

${ }^{2}$ Docente da Universidade Federal de Alfenas/MG - Departamentos de Alimentos e Medicamentos.

(*) e-mail: sinezio@unifal-mg.edu.br

Data de recebimento: 27/03/19 Aceito para publicação: 29/04/19

Persp. online: biol. \& saúde, Campos dos Goytacazes, 29 (9),43- 55, 2019

seer.perspectivasonline.com.br 


\section{INTRODUÇÃO}

A dengue é a arbovirose mais importante que afeta o ser humano, sendo uma doença febril aguda, autolimitada, com possível evolução para a forma grave (hemorrágica) e constitui sério problema de saúde pública no mundo (BRASIL, 2018).

Apesar de existir no Brasil o Plano Nacional de Combate à Dengue (PNCD) desde 2002, o Plano de Contingência Nacional para Epidemias de Dengue de 2015 e a doença estar entre as prioridades do Pacto pela Saúde desde 2006, o Brasil tem vivido sucessivas epidemias. Considerando que a Estratégia Saúde da Família (ESF) deve ser permeada pelo controle social e enfatizar a necessidade de envolvimento da população no controle da dengue (preconizado pelo PNCD) (COELHO, 2008), o monitoramento tanto de indicadores quanto da atitude do usuário dos serviços de saúde é estratégico para a diminuição de sua incidência.

Este estudo partiu do princípio de que ouvir o usuário do SUS, a respeito da qualidade do serviço de saúde e sobre sua informação sobre a dengue, é importante para a melhoria da política de combate à doença, por colaborar com uma maior adesão da população às práticas de prevenção.

Pelo fato do trabalho de campo deste estudo ter sido realizado em 2016, foram considerados aqui os dados relativos à $40^{\text {a }}$ semana de 2015 do Boletim Epidemiológico do Ministério da Saúde (BRASIL, 2015). Os dados apontaram que o mês de abril foi o de maior incidência e que no Brasil, de 2014 para 2015, o número de casos foi de 263,1 a 732,4 por 100 mil habitantes. Em relação à região sudeste e Minas Gerais, respectivamente, os casos foram de 349,4 a 1.116,3 e 269,1 a 848,9 por 100 mil habitantes. Assim, houve um aumento de incidência da ordem de 178\%, 220\% e 216\%, respectivamente para Brasil, Sudeste e Minas Gerais.

Ainda de acordo com o citado boletim epidemiológico (BRASIL, 2015), de 13 de julho de 2014 a 13 de outubro de 2015 em Minas Gerais, a ocorrência por 100 mil habitantes de casos com sinais de alarme, graves e óbitos, variaram, respectivamente de: 115 a 899, 46 a 657 e 49 a 55 . No Sudeste, esses números foram: 790 a 14.218, 282 a 6.002 e 160 a 519. Minas Gerais, portanto, apresentou melhores indicadores do que a região sudeste, especialmente em relação ao número e evolução de óbitos.

Em Minas Gerais, o sorotipo predominante em 2015 foi o DENV1 $(98,8 \%)$ e o restante $(1,2 \%)$ refere-se ao DENV2, entretanto, o DENV3, por exemplo, nos estados da Paraíba e Pernambuco, representou, respectivamente, $42,9 \%$ e 26,5\% dos casos (BRASIL, 2015). Particularmente em relação a Alfenas, a análise de dados de 2001 a 2010 produziram resultados indicativos de que as áreas de ESF tiveram menor número de casos e o maior número ocorreu no centro da cidade (NASCIMENTO, 2011). Considerado o período de 2007 a 2016, a notificação de casos suspeitos e confirmados de residentes em Alfenas foi de: 24, 4, 0, 45, 16, 20, 174, 273, 2.383 e 3.897, respectivamente em cada ano do período (BRASIL, 2019). Isso mostra que o estudo foi feito em um ano (2016) em que se vivia uma situação epidêmica da doença, o que se reverteu no ano de 2017 com apenas 35 notificações (BRASIL, 2019).

As crescentes aglomerações urbanas e o grande trânsito de pessoas são desafios para o controle da dengue, pois facilitam não apenas os criadouros do vetor, mas, também, a circulação do vírus (COELHO, 2008). Assim, é compreensível que o Plano Nacional de Combate à Dengue de 2002 enfatize que as ações devem ser de caráter permanente, dada a não evidência de erradicação do vetor em curto prazo e, também, a necessária mobilização e responsabilização da população pelo seu ambiente doméstico (COELHO, 2008). 
Tão preocupante quanto essa falta de responsabilidade pelo próprio domicílio, parece ser a negligência com o ambiente coletivo. Silva e Silva (2012), a partir de pesquisa com moradores de Boa Vista-RR, afirmam, por exemplo, que os mesmos até tinham certa preocupação com o ambiente doméstico, mas não com o coletivo.

Por outro lado, dados de pesquisa em Coromandel-MG, encontraram indicativos de que a população conhece como prevenir os criadouros do vetor, mas pouco faz a respeito em suas próprias casas (BOAVENTURA; PEREIRA, 2014). Percebe-se, portanto, que o envolvimento da população e o seu conhecimento e atitude em relação à dengue deve ser apriomorado para o controle e eliminação da doença.

Assim, este estudo teve como objetivo coletar e analisar dados sobre o nível de informação sobre dengue entre usuários da Estratégia Saúde da Família (ESF) na cidade de Alfenas-MG, Brasil.

\section{METODOLOGIA}

Trata-se de um estudo exploratório, quantitativo, descritivo e transversal, realizado no primeiro semestre de 2016 na área adscrita de 10 unidades de ESF da área urbana do município de Alfenas - MG. O público alvo foi constituído por usuários da ESF, maiores de 18 anos e com capacidade de interação cognitiva para responderem autonomamente ao questionário de pesquisa.

A amostragem foi por conveniência, entrevistando-se moradores de 10 casas vizinhas a cada ESF da área urbana de Alfenas-MG, sendo 5 no sentido centro-bairro (numeração crescente) e 5 no sentido bairro-centro (numeração decrescente), com um entrevistado por residência, somando-se 100 entrevistados. A abordagem foi a partir das casas imediatamente vizinhas à unidade de ESF, em abordagens alternadas, isto é, "casa sim, casa não". Em caso de ausência ou recusa do morador, por critério de exclusão, a casa imediatamente seguinte foi abordada e assim sucessivamente. A coleta de dados foi por meio de entrevista estruturada em questionário com questões fechadas e semi abertas.

Os dados foram tabulados e processados utilizando-se o aplicativo Microsoft Office Excel e o software livre PAST. A abordagem estatística descritiva foi feita usando-se proporções em percentual expressas em tabelas e a diferença entre proporções foi avaliada pelo teste exato de Fisher $(\mathrm{p}<0,05)$.

O Projeto de Pesquisa obedeceu às Diretrizes e Normas Regulamentadoras de Pesquisa envolvendo seres humanos, segundo a Resolução nº466/12 do Conselho Nacional de Saúde e foi aprovado pelo Comitê de Ética em Pesquisa da UNIFAL-MG sob o parecer de $\mathrm{n}^{\mathrm{o}} 1.623 .187 \mathrm{e}$ CAAE $\mathrm{n}^{\mathrm{o}}$ 57209816.9.0000.5142. As entrevistas foram realizadas após a leitura e assinatura do Termo de Concentimento Livre e Esclarecido.

\section{RESULTADOS}

A população entrevistada apresentou idade média de 44,6 anos $(\mathrm{dp}=18,1)$, e com predominância de mulheres $(65 \%)$. O número médio de pessoas por família foi de $3,3(\mathrm{dp}=1,3)$ e as famílias tiveram a seguinte distribuição por renda familiar: até 1 SM (salário mínimo) (23\%); de 1 a 3 SM (61\%); de 3 a 5 SM (12\%); de 5 a 15 SM (4\%). Considerando a renda mensal média das famílias brasileiras de 1 a 3 SM (IBGE, 2017), a maior parte da amostra mostrou-se coerente com a realidade nacional. Dos entrevistados, $18 \%$ afirmaram ter alguém com alguma doença na família no momento da entrevista. Em relação a plano de saúde suplementar, observou-se que $27 \%$ dos 
entrevistados o possuem.

De todos os entrevistados, $31 \%$ disseram ter tido dengue (por diagnóstico clínico e/ou laboratorial) em alguma fase da vida. Sobre o conhecimento dos sintomas da doença, apenas $8 \%$ disseram não conhecer. Já em relação a dengue hemorrágica, 77\% não conhecem os sintomas. Perguntados sobre como a dengue é transmitida, 78\% dos entrevistados responderam corretamente, citando o vetor ("mosquito").

A escolaridade variou de fundamental incompleto ao superior completo, com a seguinte distribuição: fundamental incompleto (23\%); fundamental completo (15\%); médio incompleto $(8 \%)$; médio completo (34\%); superior incompleto (5\%); superior completo $(15 \%)$.

A partir da Tabela 1, separando os resultados entre pessoas com menor escolaridade (fundamental incompleto, fundamental completo e médio incompleto) e com maior escolaridade (médio completo, superior incompleto e superior completo), verifica-se que $26,1 \%$ dos de menor escolaridade tiveram dengue e $35,2 \%$ dos de maior escolaridade tiveram a doença, sem diferença significativa $(\mathrm{p}=0,39)$. Mas, ao se comparar a proporção da dengue entre aqueles com nível escolar fundamental incompleto $(8,7 \%)$ com a encontrada entre os de nível superior completo (40\%), o número de casos se mostrou significativamente maior entre os de maior escolaridade $(\mathrm{p}=0,04)$. $\mathrm{O}$ mesmo acontece ao se comparar esse extremo inferior de escolaridade com aqueles com superior completo e incompleto, com proporções de $8,7 \%$ e $38,9 \%$, respectivamente $(\mathrm{p}=0,03)$.

Tabela 1: Variável sociodemográfica e casos de dengue

\begin{tabular}{|c|c|c|}
\hline \multirow[t]{2}{*}{ Variável } & \multicolumn{2}{|c|}{$\begin{array}{c}\text { Casos de dengue } \\
(\mathrm{N} \text { e } \%)\end{array}$} \\
\hline & Sim & Não \\
\hline \multicolumn{3}{|l|}{ Escolaridade } \\
\hline Fundamental Incompleto & $2(8,7 \%)$ & $21(91,3 \%)$ \\
\hline Fundamental Completo & $5(33,3 \%)$ & $10(66,7 \%)$ \\
\hline Médio Incompleto & $5(62,5 \%)$ & $3(37,5 \%)$ \\
\hline Médio Completo & $12(35,3 \%)$ & $22(64,7 \%)$ \\
\hline Superior incompleto & $1(20,0 \%)$ & $4(80,0 \%)$ \\
\hline Superior Completo & $6(40,0 \%)$ & $9(60,0 \%)$ \\
\hline \multicolumn{3}{|l|}{ Renda } \\
\hline Até $1 \mathrm{SM}$ & $4(17,4 \%)$ & $19(82,6 \%)$ \\
\hline$>1 \mathrm{SM} \mathrm{a} \leq 3 \mathrm{SM}$ & $19(31,1 \%)$ & $42(68,9 \%)$ \\
\hline$>3 \mathrm{SM} \mathrm{a} \leq 5 \mathrm{SM}$ & $6(50,0 \%)$ & $6(50,0 \%)$ \\
\hline$>5 \mathrm{SM}$ a $15 \mathrm{SM}$ & $2(50,0 \%)$ & $2(50,0 \%)$ \\
\hline Total & $31(31,0 \%)$ & $69(69,0 \%)$ \\
\hline
\end{tabular}

Apesar de em termos absolutos existir uma grande diferença na proporção de casos entre aqueles com renda familiar de até 3 salários mínimos (SM) (27\%) em relação à proporção de 50\% entre os de maior renda (acima de $3 \mathrm{SM}$ ), essa diferença não se mostrou significativa $(\mathrm{p}=0,08)$. 
Entretanto, é interessante notar que, se comparada a proporção de $17,4 \%$ da dengue entre os de menor renda familiar (até $1 \mathrm{SM}$ ) e a de $50 \%$ da dengue entre aqueles com renda de $3 \mathrm{SM}$ ou mais, o maior número de casos entre os mais ricos é significativa $(\mathrm{p}=0,04)$ (Tabela 1$)$. Resultado coerente com o fato de que dos 23 entrevistados com renda familiar de até $1 \mathrm{SM}$, apenas 1 tinha nível escolar superior completo, enquanto entre os 16 entrevistados com renda familiar acima de 3 SM, 6 tinham nível de escolaridade superior completo e 2 incompleto.

Ter ou não plano de saúde suplementar não se associou significativamente a maior ou menor número de casos $(\mathrm{p}=0,33)$. A proporção de casos também não diferiu significativamente entre os $\operatorname{sexos}(\mathrm{p}=0,37)$.

Questionados sobre a visita do agente de combate a endemias (ACE) nas residências, apenas $7 \%$ relataram não a ter recebido. O percentual de casos da doença entre quem diz ter recebido e não recebido a visita do ACE, respectivamente $31,1 \%$ e $28,6 \%$, não mostrou diferença significativa $(\mathrm{p}=0,9)$.

Em relação ao grau de preocupação com a dengue, $6 \%$ não manifestaram preocupação alguma com a doença e $70 \%$ manifestaram uma preocupação grande ou muito grande. Entre quem teve ou não dengue não houve diferença significativa em nenhum grau de preocupação com a doença (Tabela 2).

Tabela 2 - Preocupação com a dengue entre quem teve e não teve a doença.

\begin{tabular}{cccc}
\hline \multirow{2}{*}{ Grau de preocupação } & \multicolumn{2}{c}{ Teve a doença } & \multirow{2}{*}{ Valor } \\
\cline { 2 - 3 } & Sim & Não & \\
\hline Muito grande & $13(41,9 \%)$ & $21(30,4 \%)$ & 0,36 \\
Grande & $9(29,0 \%)$ & $27(39,1 \%)$ & 0,37 \\
Média & $6(19,4 \%)$ & $14(23,0 \%)$ & 1,0 \\
Pequena & $2(6,5 \%)$ & $2(2,9 \%)$ & 0,69 \\
Nenhuma & $1(3,2 \%)$ & $5(7,2 \%)$ & 0,56 \\
Total & $31(100,0 \%)$ & $69(100,0 \%)$ & \\
\hline
\end{tabular}

Também, não foi encontrada diferença significativa nos diferentes graus de preocupação com a doença entre homens e mulheres (Tabela 3). O sexo também não se apresentou como fator determinante para diferenciar significativamente quanto a: conhecimento sobre o modo de transmissão; modo de prevenção; conhecimento sobre sintomatologia da dengue clássica e hemorrágica (Tabela 3). 
Tabela 3 - Preocupação com a dengue e conhecimento sobre a doença segundo o sexo.

\begin{tabular}{|c|c|c|c|}
\hline \multirow[b]{2}{*}{ Variável } & \multicolumn{2}{|c|}{ Sexo (N e \%) } & \multirow[b]{2}{*}{ Valor $\mathbf{p}$} \\
\hline & $\begin{array}{c}\text { Feminino } \\
(65=100 \%)\end{array}$ & $\begin{array}{c}\text { Masculino } \\
(35=100 \%)\end{array}$ & \\
\hline \multicolumn{4}{|l|}{ Preocupação com a dengue } \\
\hline Muito grande & $22(33,8 \%)$ & $12(34,3 \%)$ & 1,0 \\
\hline Grande & $25(38,5 \%)$ & $11(31,4 \%)$ & 0,52 \\
\hline Média & $11(16,9 \%)$ & $9(25,7 \%)$ & 0,31 \\
\hline Pequena & $6(9,2 \%)$ & $3(8,6 \%)$ & 1,0 \\
\hline Nenhuma & $1(1,5 \%)$ & $0(0 \%)$ & 1,0 \\
\hline $\begin{array}{c}\text { Sabe como a dengue é } \\
\text { transmitida }\end{array}$ & $52(80,0 \%)$ & $26(74,3 \%)$ & 0,61 \\
\hline $\begin{array}{c}\text { Sabe como prevenir a } \\
\text { dengue }\end{array}$ & $62(95,4 \%)$ & $30(85,7 \%)$ & 0,12 \\
\hline $\begin{array}{c}\text { Conhece os sintomas da } \\
\text { dengue }\end{array}$ & $61(93,8 \%)$ & $31(88,6 \%)$ & 0,45 \\
\hline $\begin{array}{c}\text { Conhece os sintomas da } \\
\text { dengue hemorrágica }\end{array}$ & $17(26,2 \%)$ & $6(17,1 \%)$ & 0,46 \\
\hline
\end{tabular}

Das 100 pessoas entrevistadas, apenas 8 disseram não conhecer os sintomas da dengue. Foram registradas 168 citações de sintomas, sendo os mais frequentes: "febre", "dor no corpo" e "dor de cabeça", respectivamente, numa proporção de 36,9\%, 23,8\% e 17,9\% do total. Foram citados também os seguintes sintomas com respectivas proporções em relação ao total: "vermelhidão" (4,2\%), "dor" (3,0\%), "dor nos olhos" (3,0\%), "manchas" (3.0\%), "mal estar" $(1,8 \%)$, "diarreia" $(1,2 \%)$, "falta de apetite" $(1,2 \%)$, "vômitos" $(0,6 \%)$, "dor muscular" $(0,6 \%)$, "delírio" $(0,6 \%)$, "dor nas juntas" $(0,6 \%)$, "grosseira corporal desagradável" $(0,6 \%)$, "manchas na pele" $(0,6 \%)$, "manchas vermelhas" $(0,6 \%)$.

Não houve diferença significativa entre a proporção dos que disseram conhecer os sintomas da dengue clássica entre os que tiveram e os que não tiveram dengue, respectivamente $96,8 \%$ e $89,9 \%(\mathrm{p}=1,0)$.

Em relação aos sintomas da dengue clássica, embora entre todos os níveis de escolaridade existam altos índices de conhecimento a respeito (Tabela 4), os mais escolarizados (médio completo, superior incompleto e superior completo) apresentam significativamente $(\mathrm{p}=0,02)$ uma maior proporção de conhecedores $(98,1 \%)$, comparando-se com os de menor escolaridade (fundamental incompleto, fundamental completo e médio incompleto) $(84,8 \%)$.

Das 100 pessoas entrevistadas, apenas 23 disseram conhecer os sintomas da dengue hemorrágica (Tabela 4). 
Tabela 4 - Nível de escolaridade e conhecimento sobre sintomas da dengue clássica e hemorrágica

\begin{tabular}{lcc}
\hline $\begin{array}{l}\text { Nível de } \\
\text { Escolaridade }\end{array}$ & $\begin{array}{c}\text { Conhece sintomas da } \\
\text { dengue clássica } \\
\text { (N e \%) }\end{array}$ & $\begin{array}{c}\text { Conhece sintomas da } \\
\text { dengue hemorrágica } \\
\text { (N e \%) }\end{array}$ \\
\hline Fundamental Incompleto & $20(87,0 \%)$ & $3(13,0 \%)$ \\
Fundamental Completo & $11(73,3 \%)$ & $2(8,7 \%)$ \\
Médio Incompleto & $8(100 \%)$ & $2(8,7 \%)$ \\
Médio Completo & $33(97,1 \%)$ & $7(30,4 \%)$ \\
Superior incompleto & $5(100 \%)$ & $2(8,7 \%)$ \\
Superior Completo & $15(100 \%)$ & $7(30,4 \%)$ \\
Total & $92(92 \%)$ & $23(23 \%)$ \\
\hline
\end{tabular}

Todos os sintomas de dengue hemorrágica citados relacionaram-se com sangramento, como, por exemplo, "manchas vermelhas e sangramento" e "hemorragia, dor e febre". Embora os mais escolarizados (médio completo, superior incompleto e superior completo) apresentem uma maior proporção de conhecedores a respeito dos sintomas da forma hemorrágica (29,6\%), comparando-se com os de menor escolaridade (fundamental incompleto, fundamental completo e médio incompleto) $(15,2 \%)$, essa diferença não se mostrou significativamente diferente $(\mathrm{p}=0,10)$.

Porém, há significativamente maior conhecimento sobre os sintomas da dengue hemorrágica entre os de maior escolaridade $(\mathrm{p}=0,03)$ (quando comparados os extremos de escolaridade), sendo que $30,4 \%$ daqueles com superior completo afirmaram conhecer os sintomas, enquanto apenas $13,0 \%$ daqueles com fundamental incompleto disseram conhecê-los.

Entre os de maior escolaridade (superior completo, superior incompleto e médio completo) $35,2 \%$ disseram possuir plano de saúde suplementar, quantidade significativamente maior do que os $15,2 \%$ que disseram não ter entre os de menor escolaridade $(\mathrm{p}=0,02)$.

A partir dos dados da Tabela 5, verifica-se que os de maior escolaridade (médio completo, superior incompleto e superior completo) significativamente sabem mais sobre a prevenção da dengue $(98,1 \%)$ do que os de menor escolaridade (fundamental incompleto, fundamental completo e médio incompleto) $(84,8 \%)(\mathrm{p}=0,02)$. 
Tabela 5 - Nível de escolaridade e conhecimento sobre modo de transmissão e prevenção da dengue

\begin{tabular}{lcc}
\hline Nível de Escolaridade & $\begin{array}{c}\text { Sabe como a dengue é } \\
\text { transmitida }\end{array}$ & $\begin{array}{c}\text { Sabe como prevenir a } \\
\text { dengue }\end{array}$ \\
\hline Fundamental Incompleto & $18(78,3 \%)$ & $23(100 \%)$ \\
Fundamental Completo & $8(69,6 \%)$ & $10(66,7 \%)$ \\
Médio Incompleto & $5(87 \%)$ & $6(75,0 \%)$ \\
Médio Completo & $28(73,9 \%)$ & $34(100 \%)$ \\
Superior incompleto & $4(95,7 \%)$ & $5(100 \%)$ \\
Superior Completo & $15(100 \%)$ & $4(93,3 \%)$ \\
\hline
\end{tabular}

Observa-se também que, apesar das epidemias ocorridas local, regional e nacionalmente, ainda $22 \%$ dos entrevistados disseram não saber ser um mosquito o transmissor da dengue. Porém, entre os de maior escolaridade (médio completo, superior incompleto e superior completo) apenas $13,0 \%$ disseram não saber a respeito, proporção significativamente menor $(\mathrm{p}=0,03)$ do que os $32,6 \%$ dos de menor escolaridade (fundamental incompleto, fundamental completo e médio incompleto) que disseram não saber que o mosquito é o vetor da doença (Tabela 5).

Em termos de "maior fonte de informação sobre a dengue" foram observados os seguintes resultados: TV (91\%), profissionais de saúde (24\%), internet (18\%), rádio (10\%), jornal (7\%), vizinhos (7\%), livros (2\%). Os resultados referem-se tanto à citação exclusiva de uma determinada fonte quanto à sua citação junto a uma ou mais de uma fonte.

\section{DISCUSSÃO}

A significativa maior proporção de dengue entre os de maior escolaridade contraria a suposição de que pessoas mais escolarizadas teriam nível de informação suficiente para melhor prevenir a doença. Uma especulação a ser feita é que se a maior escolaridade estiver relacionada à maior renda e isto condicionar uma maior mobilidade geográfica às pessoas (trabalho, passeios, turismo, etc), pessoas nesta situação poderiam estar se expondo mais a ambientes com maior infestação do vetor. Outra hipótese plausível para explicar o maior número de casos entre os de maior escolaridade seria a de que teriam maior acesso a meios de confirmação diagnóstica (exames laboratoriais, por exemplo), por terem maior renda e mais plano de saúde suplementar do que os de menos escolaridade. No entanto essa hipótese é questionável pelo fato de que ter ou não plano de saúde suplementar não se associou significativamente a um maior ou menor número de casos de dengue.

Coerente com a maior proporção da doença entre os mais escolarizados, observou-se que foi também entre os entrevistados de famílias com maior renda que o número de casos foi mais alto. Em um estudo com a população de Alfenas-MG, observou-se que a dengue esteve presente tanto 
nas regiões com melhor perfil socioeconômico, quanto em áreas piores em saneamento ambiental, condições de renda, escolaridade e adensamento populacional (NASCIMENTO, 2011). Nesse sentido, e pelos resultados aqui expostos, os dados contrariam a ideia de que maior renda e escolaridade, por si só, seriam um fator de proteção contra a doença, indicando que o grau e a qualidade da informação sobre a dengue são fundamentais para o seu controle.

Lenizi e Coura (2004) ressaltam que a informação é um importante componente nos programas de promoção da saúde e prevenção de doenças, e que mensagens informativas deveriam circular intensivamente durante todo o ano, evitando, inclusive, a falsa ideia de que dengue só ocorre no verão. Os autores ressaltam, ainda, que campanhas de combate à dengue no Brasil não são de caráter permanente, tendo sua retomada e intensificação à época do verão, podendo assim ser umas das causas da falta de informação e conhecimento dos sintomas.

Além disso, o uso inadequado das informações pode estar atrelado ao grau de escolaridade dos sujeitos da pesquisa, o que pode dificultar o entendimento da importância das medidas saneantes de controle do Aedes aegypti (SILVA et al., 2015). Pertinente a este aspecto, este estudo encontrou diferença significativa quanto à relação entre nível de escolaridade e a resposta positiva quanto a saber como prevenir a dengue. Os mais escolarizados significativamente manifestaram maior informação sobre prevenção do que os menos escolarizados.

De toda forma, os resultados aqui apresentados, indicam que diminuir o custo de informação por si só, em tese pela maior escolaridade, pode não ser suficiente para mudanças comportamentais que diminuam risco. O papel das relações sociais na determinação comportamental pode ser uma variável importante a ser considerada. Segundo Silva et al. (2015), embora exista uma relação direta entre o conhecimento sobre dengue e atitudes preventivas de proteção, as medidas contra mosquitos adultos são provavelmente usadas somente quando as pessoas vivenciam o problema. Assim, ressaltam os autores, fechar a lacuna entre conhecimento e prática continuará sendo um desafio importante para o controle da doença. É importante também considerar que o conhecimento a ser aplicado não deve apenas derivar do discurso técnico e informação especializada, mas ser apreendido do conhecimento prático construído pela própria população (SOUZA et al., 2018).

Pensando-se na prevenção em nível secundário, do ponto de vista da história natural da doença, o reconhecimento dos sintomas é fundamental para a terapêutica e vigilância clínica. Assim, é relevante que apenas $8 \%$ disseram não conhecer os sintomas da dengue, mas $77 \%$ não souberam citar sintomas da forma hemorrágica. Trata-se de um resultado especialmente preocupante pelo possível desfecho fatal que a doença pode ter nessa forma mais grave, quando o tratamento de suporte ou o suporte hemodinâmico não é adotado oportunamente. Para tanto pode contribuir a falta da auto percepção de sinais e sintomas da forma hemorrágica. Gonçalves (2015) também observou, a partir de estudo sobre o efeito de campanhas nas escolas sobre o conhecimento dos pais, que o conhecimento sobre a dengue clássica foi maior do que sobre a hemorrágica.

Não houve diferença significativa da proporção dos que disseram conhecer os sintomas da dengue clássica entre os que tiveram e os que não tiveram dengue, indicando que a informação ou interpretação de terceiros pode ser tão ou mais importante do que a própria experiência a respeito. Souza (2012) também observou que em relação aos sinais e sintomas de dengue, o conhecimento da população foi considerado bom, se levados em conta sinais inespecíficos como a febre, cefaleia e mialgia.

No que se refere à correta citação de sintomas da dengue hemorrágica, os dois estratos de escolaridade não apresentaram diferença. Considerando-se, porém, os extremos (fundamental

Persp. online: biol. \& saúde, Campos dos Goytacazes, 29 (9),43- 55, 2019

seer.perspectivasonline.com.br 
incompleto e superior completo) houve significativamente maior conhecimento de tais sintomas entre os mais escolarizados. Isso não é de surpreender, pois considerando que pessoas com maior escolaridade processam melhor a informação de modo lógico ou relacional, ter nível superior completo contribuiria muito para supor a resposta a partir da própria pergunta feita "você sabe quais os sintomas da dengue hemorrágica?".

Também o conhecimento sobre o modo de transmissão foi significativamente maior entre aqueles do estrato de maior escolaridade. Nesse sentido, estudo de Boaventura e Pereira (2014) observou que o nível de acerto dos entrevistados que possuíam ao menos o Ensino Médio Completo em relação à sintomatologia, modo de transmissão e necessidade de procurar assistência médica especializada foi significativamente maior, quando comparado com os entrevistados que apresentavam apenas Educação Básica incompleta.

$\mathrm{Na}$ atualidade a televisão desempenha papel fundamental de comunicação social, sendo que a grande maioria (91\%) a citou como principal fonte de informação sobre dengue. Entretanto, isso não foi suficiente para que se pudesse associar maior informação à prevenção. Além disso, embora constando como segunda principal fonte informativa, os profissionais de saúde foram citados por apenas $24 \%$ dos entrevistados. Isso chama a atenção para a necessidade de maior inserção de toda a equipe da ESF no esforço de prevenção da doença e, especialmente, um maior e mais qualificado protagonismo do agente comunitário de saúde (ACS), por ser fundamental às suas responsabilidades funcionais o contato cotidiano e proativo com a comunidade.

Há muito se ressalta que a principal forma de prevenção da dengue é a drástica redução da população do mosquito transmissor, aliando intimamente a vigilância entomológica e epidemiológica no controle do vetor (TEIXEIRA, 1999). Assim, saber sobre a efetividade da visita não só do ACS, mas do ACE, é de fundamental importância. O número de casos da doença entre quem diz ter recebido ou não a visita do ACE não mostrou diferença significativa. Isso pode significar tanto que a infecção não se deu no ambiente doméstico (a presença de focos do mosquito nos domicílios dos infectados não seria significativa) ou que a educação em saúde e orientação preventiva não foi efetiva e oportuna. Nesse sentido, cabe questionar se o fato de a grande maioria (93\%) ter recebido a visita do ACE é reflexo de uma ação de vigilância mais rotineira ou está associada à tentativa de controle da epidemia então vivida pelo município no ano da pesquisa (2016).

Um possível complicador para a efetividade da ação tanto do ACE quanto do ACS é justamente a imprecisão e deficiente interação do papel desses profissionais. Souza et al.. (2018) encontraram resultados indicativos nesse sentido, inclusive, e de modo mais preocupante, o sentimento de desobrigação dos ACS em relação ao controle vetorial. Por outro lado, a incorporação das ações preconizadas pelo Programa Nacional de Combate à Dengue à rotina dos ACS deve ser muito bem equacionada, para que não comprometa a efetividade das demais atribuições da ESF (CAZOLA et al., 2014).

Embora muitas pesquisas sobre dengue tenham foco em mulheres, por serem mais frequentemente as principais responsáveis pelos cuidados com o ambiente doméstico (GOTO, 2014), o presente estudo não encontrou significativa diferença entre os sexos no grau de preocupação ou nível de informação sobre a doença. Isso, aliado aos demais resultados encontrados, indica que é difícil evidenciar uma prioridade de público a ser considerado para maior esclarecimento, sendo necessários esforços junto a todos os segmentos e que valorizem uma dinâmica comunitária a respeito. De fato, alguns autores ressaltam que o poder público precisa

Persp. online: biol. \& saúde, Campos dos Goytacazes, 29 (9),43- 55, 2019 
melhorar práticas de educação em saúde para aumentar o envolvimento da comunidade, considerando-a menos como um "paciente" a receber prescrições e mais como um sujeito decisivo na produção de respostas mais efetivas (SOUZA et al., 2018; MATOS et al., 2014).

\section{CONCLUSÕES}

A maioria das pessoas entrevistadas mostrou conhecimento a respeito dos sintomas da dengue clássica, sobre como prevenir a doença e seu modo de transmissão. Embora a forma mais grave da dengue seja denominada de "hemorrágica", e este termo tenha sido citado nas entrevistas, uma minoria disse saber sobre os sintomas dessa manifestação clínica. Ou seja, nem por efeito indutivo os entrevistados citaram a hemorragia como sintoma.

A maior ou menor proporção da dengue entre as pessoas entrevistadas não se mostrou um fator diferenciador sobre o grau de preocupação e nível de conhecimento sobre os sintomas da doença, o que pode indicar uma boa circulação de informação a respeito destes aspectos. Também, a maior ou menor preocupação e informação sobre a doença não teve diferença significativa entre os sexos.

Mais interessante ainda, e que merece maior atenção de pesquisa e melhores estratégias de prevenção, é que o fato de os mais escolarizados terem significativamente maior informação sobre a dengue não parece ter sido suficiente para que eles melhor prevenissem a doença. Foi entre os mais escolarizados e com maior renda familiar que se observou o maior número de casos da dengue.

Por tratar-se de um estudo exploratório e sem grupo controle (não usuários da ESF), os dados apresentados não permitem uma conclusão mais definitiva sobre a efetividade dos profissionais da ESF sobre o número de casos na população adscrita. Mas, é preocupante a pequena proporção dos que disseram conhecer os sintomas da forma hemorrágica da doença $(23 \%)$ e a proporção (24\%) em que foi indicado o "profissional de saúde" como fonte principal de informação sobre a dengue. A televisão se mostrou como principal fonte de informação (91\%), o que é importante, mas um maior protagonismo da equipe da ESF seria desejável e é fundamental.

Os profissionais médicos e de enfermagem são estratégicos não apenas para conscientizar sobre os riscos da dengue hemorrágica, mas sobre medidas de prevenção individual e não apenas domiciliar. Destaque-se, também, que um maior protagonismo do ACS seria de grande relevância, tanto através de um maior envolvimento imediato com o controle da doença, quanto sendo alvo de um crescente aprimoramento técnico em comunicação social a respeito do tema. Esse aprimoramento, certamente, também é importante para o ACE, mas a presença deste último associase a uma ação mais sazonal e, razoável supor, mais provavelmente sob a lógica mais restrita do modelo campanhista do que sob a orientação mais ampla do modelo de vigilância em saúde.

\section{REFERÊNCIAS}

BRASIL. Ministério da Saúde. Dengue, descrição da doença. Brasília; 2018. Disponível em: < http://portalms.saude.gov.br/saude-de-a-z/dengue/descricao-da-doenca>. Acesso em: 17 jan. 2018.

COELHO, G.E. Dengue: desafios atuais. Secretaria de Vigilância em Saúde. Programa Nacional de Controle de Dengue. Epidemiol Serv Saúde, v. 17, n. 3, p. 231-233, 2008.

BRASIL. Ministério da Saúde. Secretaria de Vigilância em Saúde. Monitoramento dos casos de dengue e febre de chikungunya até a Semana Epidemiológica 40. Bol Epidemiol., v. 46, n. 33, 2015 . 
NASCIMENTO, M. C. Geoepidemiologia da dengue no município de Alfenas, MG. 2011. 70 p. Dissertação (Mestrado em Saúde na Comunidade) - Faculdade de Medicina - Universidade de São Paulo, Ribeirão Preto, 2011.

BRASIL. Ministério da Saúde. Departamento de Informática do SUS - DATASUS. Informações de Saúde, Epidemiológicas e Morbidade: Doenças e Agravos de Notificação - De 2007 em diante (SINAN). Disponível em: < http://tabnet.datasus.gov.br/cgi/tabcgi.exe?sinannet/cnv/denguebr.def> e < http://tabnet.datasus.gov.br/cgi/tabcgi.exe?sinannet/cnv/denguebbr.def> Acesso em 14 abr. 2019.

SILVA, L. C.; SILVA, R. D. Percepção ambiental dos moradores da área Conjunto Cidadão e a relação com os casos de dengue no bairro Senador Hélio Campos, Boa Vista-RR. Rev Norte Cient., v. 7, n. 1, p. 139-150, 2012.

BOAVENTURA, P. D.; PEREIRA, B. B. Análise da relação entre conhecimentos e atitudes da população de Coromandel, Minas Gerais, Brasil, acerca da dengue. Hygeia, v. 10, n. 18, p. 121-128, 2014.

LENZI, M. F.; COURA, L. C. Prevenção da dengue: a informação em foco. Rev Soc Bras Med Trop. v. 37, n. 4, p. 343-350, 2004.

SILVA, G. M. et al. Percepções e atitudes sobre a dengue dos usuários do sistema único de saúde no município de Aracaju, Sergipe, Brasil. Rev APS. v. 18, n. 3, p. 341-353, 2015.

SOUZA, K. R. et al. Saberes e práticas sobre controle do Aedes aegypti por diferentes sujeitos sociais na cidade de Salvador, Bahia, Brasil. Cad. Saúde Pública. v. 34, n. 5, e00078017, 2018.

GONÇALVES, R. P. et al. Contribuições recentes sobre conhecimentos, atitudes e práticas da população brasileira acerca da dengue. Saúde Soc. v. 24, n. 2, p. 578-593, 2015.

SOUZA, V. M. M. Avaliação do conhecimento, atitudes e práticas sobre dengue no Município de Pedro Canário. Rev Panamazonica Saude. v. 3, n. 1, p. 37-43, 2012.

TEIXEIRA, M. G.; BARRETO, M. L. Guerra Z. Epidemiologia e medidas de prevenção do Dengue. Inf Epidemiol Sus. v. 8, n. 4, p. 5-33, 1999.

CAZOLA, L. H. O. et al. Incorporação das atividades de controle da dengue pelo agente comunitário de saúde. Rev Saúde Pública. v. 48, n. 1, p. 113-122, 2014.

GOTO, L. F. Crenças sobre a dengue entre residentes do município de São Paulo e o impacto na campanha do governo de combate à doença. 2014. 98 p. Monografia (trabalho de Conclusão de Curso em Administração de Empresas) - Faculdade de Economia, Administração e Contabilidade Universidade de São Paulo, São Paulo, 2014.

MATOS, A. P. C. et al. Do conhecimento à ação: o enfoque da Ecossaúde no contexto da dengue a partir de uma experiência de educação popular. Rev. Bras. Pesq. Saúde. v. 16, n. 4, p. 63-71, 2014. 